\title{
On the nature of cosmic rays above the Greisen-Zatsepin-Kuz'min cut off
}

\author{
L. A. Anchordoqui, M. Kirasirova, T. P. McCauley, \\ S. Reucroft, and J. D. Swain \\ Department of Physics, Northeastern University, Boston, MA 02115
}

\begin{abstract}
A re-examination of the atmospheric cascade profile of the highest energy cosmic ray is presented. The study includes air-shower simulations considering different cross sections, particle multiplicity and variation of the hadronic-event-generator to model interactions above $200 \mathrm{GeV}$. The analysis provides evidence that a medium mass nucleus, say ${ }^{41} \mathrm{Ca}$, resembles the shower profile quite well (in agreement with previous studies). This result does not support the idea, increasingly popular at present, that the highest energy particles are protons, derived from the decay of supermassive relic particles. On the other hand, we show that debris of relativistic super-heavy nuclei, which can survive a $100 \mathrm{Mpc}$ journey through the primeval radiation are likely to generate such a kind of cascade.
\end{abstract}

PACS number(s): 96.40.Tv, 13.85.Tp, 96.40Pq.

Key words: Cosmic rays, chemical composition, air-shower simulation 
Shortly after the microwave background radiation (MBR) was discovered [1], it became clear that ultra high energy cosmic rays (CRs) undergo reactions with the relic photons yielding a steep drop in their energy attenuation length. Therefore, if the CR sources are all at cosmological distances, the energy spectrum would exhibit a Geisen-Zatsepin-Kuz'min (GZK) cut off slightly above $100 \mathrm{EeV}\left(1 \mathrm{EeV} \equiv 10^{18} \mathrm{eV}\right)[2]$. The spectral cut off is less sharp for nearby sources, viz., distances $<100 \mathrm{Mpc}$.

Over the last few years, several giant air showers have been detected, which imply the arrival of "super-GZK" particles [3]. Namely, the Haverah Park experiment reported several events with energies near or slightly above 100 $\mathrm{EeV}$ [4]. On October 15, 1991 the Fly's Eye experiment recorded the highest energy cosmic ray ever detected on Earth $(E \sim 300 \mathrm{EeV})$ [5]. $\square$ More recently, the Akeno Giant Air Shower Array (AGASA) has reported several remarkable $\mathrm{CR}$ events scattered across half the sky dramatically confirming that the CR spectrum does not end with the GZK cut off [7].

Many theories have been suggested to explain the origin and nature of these particles. At the most general level, the production mechanisms can be classified into two distinct groups: "bottom-up" acceleration arena, and "top-down" decay scenario, each of them branching out into various classes.

The "bottom-up" mechanism usually implies the stretching of rather well known acceleration processes to their theoretical limits (and sometimes beyond). It involves particle acceleration in the accreation flows of cosmological structures. Examples are one-shot acceleration in active galactic nuclei (AGNs) [8], large scale shocks resulting from structure formation [9], acceleration in quasar remnant cores of nearby giant elliptical galaxies [10], collisions of galaxies [11], acceleration in catastrophic events associated with gamma ray bursts [12], and acceleration in hot spots of extragalactic radio sources [13].

On the other hand, the "top-down" scenario (mischievously) escapes from the acceleration problem by assuming that charged and neutral primaries simply arise by quantum mechanical decay of supermassive elementary $X$ particles $\left(m_{X} \sim 10^{25}-10^{28} \mathrm{eV}\right)$ [14]. Sources of these exotic particles at present could be topological defects (TDs) left over from early universe phase transitions associated with spontaneous symmetry breaking underlying unified models of high energy interactions. Due to their topological stability, the TDs (magnetic monopoles, cosmic strings, domain walls, etc.) can survive forever with $X$ particles trapped inside them. Nevertheless, from time to time, some TDs can be destroyed through collapse, annihilation, or other processes, and the energy stored is released in the form of massive quanta that would typically decay into quarks and leptons. The subsequent hadronization of the quarks would

$\overline{1}$ Near the arrival direction of this event the Yakutsk experiment detected an extensive air shower whose energy may also reach $300 \mathrm{EeV}[6]$. 
produce jets of hadrons containing mainly light mesons (pions) together with a small fraction (3\%) of nucleons. Thus, the "top-down" injection spectra would be dominated by gamma rays and neutrinos originated by pion decays.

There have been arguments for some time that the cosmic photon background makes the universe opaque to ultra high energy gamma-rays [15]. The most efficient target for these rays to produce $e^{+} e^{-}$pairs are background photons of energy $\sim m_{e}^{2} / E \leq 10^{-6} \mathrm{eV} \approx 100 \mathrm{MHz}$. The mean free path for a $100 \mathrm{EeV}$ (1000 EeV) photon to annihilate on the radio background is believed to be $10-40 \mathrm{Mpc}(1-5 \mathrm{Mpc})[16,17]$. Consequently, the measurement of the diffuse gamma-ray background imposes several constraints on the total amount of electromagnetic energy density injected and recycled by cascading to lower energies, yielding bounds on TD injection spectra [18]. It is also noteworthy that the expected high diffuse neutrino flux above $1 \mathrm{EeV}$ sets additional bounds on the "top-down" decay scenario [19].

The identity of the primary particle certainly deepens the mystery. Strictly speaking, a $100 \mathrm{EeV}$ proton traverses a nearly straight line through the galactic or extragalactic magnetic fields and yet no compelling local sources have been identified within the expected scattering cone of the highest energy event [20]. Furthermore, the atmospheric shower profile is reported by Fly's Eye [5] to roughly fit that of a proton, or a gamma-ray that peaks somewhat late in theatmosphere [16]. It is also reported that a medium mass nucleus seems to fit this shower profile.

Heavy nuclei have their own merits. They can be accelerated to higher terminal energies because of their larger charge. Additionally, a heavy nucleus would have a larger scattering cone since they can be deflected more by the magnetic fields on the way to the observer. However, there are still other problems left in interpreting all the "super-GZK" events as heavy nuclei: It has long been known that above $200 \mathrm{EeV}$ nuclei should be photodissociated by the MBR within a few Mpc [21], and there are few nucleus-emitting-sources in the neighborhood of the Earth. More speculative primaries have also been proposed, such as dust grains [22] or even neutrinos [16]. On the one hand, the grains turn out to be unstable with respect to the development of a fracture. The path length up to the first break-up turns out to be much less than the characteristic size of the Milky Way [23]. In addition, the depth of shower maxima registered by the Haverah Park and Volcano Ranch experiments do not correspond to the expected picture of showers initiated by relativistic specks of dust [24]. Neutrinos, on the other hand, can traverse unscathed through the primeval radiation, but they would predominantly give rise to deeply penetrating showers in the atmosphere, again, in disagreement with observations. Putting all these together one can naïvely say that aside from some nucleus species, the broad phylum of particles does not seem to fit the cascade development of the highest energy event. 
All in all, the determination of the chemical composition appears crucial in discriminating between the possible origin of the highest energy cosmic rays. In light of this, we decided to re-examine the shower profile reported by the Fly's Eye experiment. In this letter we present an extension of the previous analysis [16] studying the effects of different atmospheric-cascade-models and parameter values.

The Fly's Eye observes an air shower as a nitrogen fluorescence light source which moves at the speed of light along the path of a high energy particle traversing the atmosphere [25]. In other words, it directly detects the longitudinal development of the air cascade. The simulation of the shower evolution depends sensitively on the first few interactions, necessarily related to the quality of our "understandying" of hadronic collisions. Soft multiparticle production with small transverse momenta with respect to the collision axis is a dominant feature of most events in high energy hadronic interaction. Despite the fact that strict calculations based on ordinary QCD perturbation theory are not feasible, some phenomenological approaches successfully take into account the main properties of soft diffractive processes [26,27]. In these models, the interactions are no longer described by single particle exchange, but by highly complicated collective modes known as Reggeons [28]. The slow growth of the cross section with the centre of mass energy requires a dominant contribution of a special Reggeon, the Pomeron [29]. As a result of the diffractive interactions, constitutents get excited and produce particles, which, in the fragmentation phase, are modelled by strings [30]. The physical picture at super-high energies is quite different. Interactions are not fully described within the framework of standard Gribov-Regge theory [28]. At these extremely high energies, appart from the usual excitations of the participants, hard parton-parton collissions (between the beam and target hadrons) take place. A suitable scenario for the joint description of both, soft and semihard hadronic physics has yet to see the light of day. The well known codes QGSJET [31] and SIBYLL [32] represent two of the best simulation tools to model hadronic interactions at the highest energies.

The underlying idea behind SIBYLL is that the increase in the cross section is driven by the production of minijets [32]. The probability distribution for obtaining $N$ jet pairs (with $p_{\mathrm{T}}^{\text {jet }}>p_{\mathrm{T}}^{\min }$, being $p_{\mathrm{T}}^{\min }$ a sharp threshold on the transverse momentum below which hard interactions are neglected) in a collision at energy $\sqrt{s}$ is computed regarding elastic $p p$ or $p \bar{p}$ scattering as a difractive shadow scattering associated with inelastic processes. The algorithms are tuned to reproduce the central and fragmentation regions data up to $p \bar{p}$ collider energies, and with no further adjustments they are extrapolated several orders of magnitude. On the other hand, in QGSJET the theory is formulated entirely in terms of Pomeron exchanges. The basic idea is to replace the soft Pomeron by a so-called "semihard Pomeron", which is defined to be an ordinary soft Pomeron with the middle piece replaced by a QCD parton 
ladder. Thus, minijets will emerge as a part of the "semihard Pomeron", which is itself the controlling mechanism for the whole interaction [31]. The different aproaches used in both codes to model the underlying physics show up clear differences in multiplicity predictions which increase with rising energy [33]. It is important to stress that although the differences seem to become washed out as the shower front gets closer to the ground, the footprints of the first hadronic colissions are still present in the longitudinal development. Consequently, the first generation of particles plays a paramount role in determining the chemical composition of the "super-GZK" event reported by Fly's Eye.

In order to determine the progenitor of the highest energy event, we carried out a Monte Carlo simulation of the atmospheric shower profile using the AIRES program (version 2.2.1) [34]. The simulation includes interaction with both the Earth magnetic field and nuclei in the atmosphere. We have taken into account possible representative variations in the transport code, cross sections, particle multiplicity and hadronic models by using SIBYLL and QGSJET to generate interactions above $200 \mathrm{GeV}$.

We generated several sets of nucleus showers with the mass equally spread in the AIRES range (up to ${ }^{56} \mathrm{Fe}$ ). In addition, air-showers of superheavy nuclei were simulated following the superposition model. This model assumes that an average shower produced by a nucleus with energy $E$ and mass number $A$ is indistinguishable from a superposition of $A$ proton showers, each with energy $E / A$. The particles were injected at the top of the atmosphere $(100 \mathrm{~km} . \mathrm{a} . \mathrm{s} . \mathrm{l})$ with zenith angle $43.9^{\circ}$, and the magnetic field was set to reproduce that prevailing upon Fly's Eye experiment [35]. All shower particles with energies above the following thresholds were tracked: $750 \mathrm{keV}$ for gammas, $900 \mathrm{keV}$ for electrons and positrons, $10 \mathrm{MeV}$ for muons, $60 \mathrm{MeV}$ for mesons and $120 \mathrm{MeV}$ for nucleons and nuclei. The results of these simulations were processed with the help of the AIRES analysis programs.

The data analysis is performed by means of a $\chi^{2}$ test [36]. We assume that the set of measured values by Fly's Eye are uncorrelated, i.e, any depth measurement is independent of each other. Then, we make use of the quantity

$$
\chi^{2} \equiv \sum_{j=1}^{N} \frac{\left|x_{j}-\alpha_{j}\right|^{2}}{\sigma_{x_{j}}^{2}},
$$

where $N$ is the total number of points in the analysis, $\sigma_{x_{j}}$ is the error on the $x_{j}$ th coordinate, $x_{j}$ is the measured value of the coordinate, and $\alpha_{j}$ the (hypothetical) true value of the coordinate. Generally speaking, the simulated shower profile would give a good representation of the data if the $\chi^{2}$ is roughly equal to the number of data points, i.e., $\chi^{2} \approx 12$. The results for different primaries are listed in Table I. 
Table 1

$\chi^{2}$ for different primaries

\begin{tabular}{|c|c|c|}
\hline Primary & $\chi_{\text {SIBYLL }}^{2}$ & $\chi_{\text {QGSJET }}^{2}$ \\
\hline$\gamma$ & 1159.79 & 1105.31 \\
\hline$p$ & 413.87 & 324.89 \\
\hline${ }^{16} \mathrm{O}$ & 197.18 & 36.75 \\
\hline${ }^{17} \mathrm{O}$ & 191.38 & 19.56 \\
\hline${ }^{18} \mathrm{O}$ & 209.11 & 27.29 \\
\hline${ }^{27} \mathrm{Al}$ & 109.77 & 22.65 \\
\hline${ }^{32} \mathrm{~S}$ & 125.80 & 17.92 \\
\hline${ }^{33} \mathrm{~S}$ & 92.82 & 62.94 \\
\hline${ }^{38} \mathrm{Ar}$ & 119.03 & 51.68 \\
\hline${ }^{39} \mathrm{~K}$ & 110.82 & 17.05 \\
\hline${ }^{40} \mathrm{Ca}$ & 126.11 & 57.02 \\
\hline${ }^{41} \mathrm{Ca}$ & 78.46 & 12.54 \\
\hline${ }^{42} \mathrm{Ca}$ & 79.47 & 90.87 \\
\hline${ }^{43} \mathrm{Ca}$ & 126.00 & 24.24 \\
\hline${ }^{46} \mathrm{Ti}$ & 116.76 & 38.40 \\
\hline${ }^{47} \mathrm{Ti}$ & 87.98 & 96.70 \\
\hline${ }^{56} \mathrm{Fe}$ & 71.45 & 72.50 \\
\hline${ }^{84} \mathrm{Kr}$ & 71.49 & 232.60 \\
\hline${ }^{108} \mathrm{Ag}$ & 102.11 & 91.16 \\
\hline${ }^{131} \mathrm{Xe}$ & 15.40 & 38.04 \\
\hline${ }^{195} \mathrm{Pt}$ & 30.99 & 87.70 \\
\hline${ }^{197} \mathrm{Au}$ & 41.88 & 426.06 \\
\hline${ }^{200} \mathrm{Hg}$ & 18.63 & 515.50 \\
\hline${ }^{207} \mathrm{~Pb}$ & 19.03 & 298.01 \\
\hline${ }^{238} \mathrm{U}$ & 19.94 & 349.81 \\
\hline
\end{tabular}

Definite conclusions on the chemical composition cannot be reached, mainly because of large fluctuations from model to model. Without belaboring the point, the main differences between the hadronic-event-generators can be ascribed to two different parameters: the inelastic cross section, and the energy fraction of the parent particle converted into the production of secondary par- 
ticles, the so-called "inelasticity". In order to match collider data, the brutal truncation of the soft hadronic processes (SIBYLL) enforces interactions with a large average fraction of the energy going into the leading baryon (elasticity) and a small fraction into secondaries (inelasticity) than those governed by QGSJET. As a consequence, SIBYLL's showers require more generations of particles undergoing hadronic collisions, yielding a delay in the electromagnetic shower development (strongly correlated with $\pi^{0}$ decays). However, both the cross section, and the inelasticity seem to affect the shower development in a similar fashion since increasing inelasticity as well as cross section lead to faster cascades. Therefore, primaries with very different combination of cross section and inelasticity are able to describe the given longitudinal development.

In particular, the large elasticity of SIBYLL leads to a monotonically decreasing behavior of the $\chi^{2}$ (with increasing primary mass) for the AIRES nucleus interval, $A<56$. Another feature to point out are small fluctuations that appear around sulfur and calcium when considering different isotopes, as seen in Fig. 1. When the showers are approximated by the superposition model $(A>56)$, the $\chi^{2}$ shows larger fluctuations. Although for ${ }^{131} \mathrm{Xe}$, and ${ }^{200} \mathrm{Hg}$ the $\chi^{2}$ approaches rather reasonable values, the reader should keep in mind the crudeness of present shower simulations. In the simulations with QGSJET+AIRES, the $\chi^{2}$ reaches a minimum at ${ }^{41} \mathrm{Ca}$, leading to a plain representation of the shower profile (see Fig. 2). In this case the evolution of the atmospheric cascade reveals a complex nuclear structure. Around the minimum, the $\chi^{2}$ behavior depends on the ratio between neutrons and protons. The same effect is present (although with shifted $\chi^{2}$ ) in oxygen induced showers (the reader is referred again to Fig. 1). The point to be made is this: Even though the primary chemical composition remains hidden by the hadronic interaction model, it is evident that in both cases the shower is completely inconsistent with a proton, or a gamma-ray primary.

Let us end by reviewing the current observational status, and discussing the nature of the highest energy CRs.

- Up to now, the AGASA experiment reported 7 events above $100 \mathrm{EeV}$. Large shower to shower fluctuations make it difficult to get a definite dependence of composition from muon density at $1000 \mathrm{~m}$ from the shower core (see Fig. 3 of Ref. [37]). The situation is further complicated if we take into account that not only two mass components (iron-proton) are allowed, but that many $A$-values could be present. There is no candidate shower of gammaray primary in this sample [37].

- The density profile of the highest energy event detected by the Yakutsk experiment is almost dominated by muons, which argues against proton/gammaray initiation [6].

- The atmospheric profile of the highest energy cosmic ray is inconsistent with that initiated by a proton or a gamma-ray. Moreover, an analysis of 
the frequency distribution of the depth maximum values recorded by Fly's Eye, indicates that in the energy range $3-10 \mathrm{EeV}$, there is a significant fraction of nuclei with charge greater than one [38].

Whatever the source(s) of the highest energy cosmic rays, because of their interactions with magnetic fields, and also with the universal diffuse radiation backgrounds permeating the universe, the energy spectra, composition and arrival directions are affected by propagation. In particular, in the case of heavy nuclei, the composition of the arriving particles may differ substantially from that of the source. For instance, an iron nucleus which is injected into the intergalactic medium with a Lorentz factor of $\gamma_{0}=4 \times 10^{9}$, after propagating $\approx 100 \mathrm{Mpc}$ will end up with $A \approx 302^{2}$ Such metamorphosis is the result of two basic interactions: photodisintegration and pair creation. The latter reduces the Lorentz factor significantly during propagation avoiding the complete disintegration of the nucleus. Stecker and Salamon have pointed out that a heavy nucleus emitted by the strong radio galaxy NGC315, which is 60 Mpc away and within the scattering cone of the highest energy event detected by AGASA, would have an energy cut off $\approx 130 \mathrm{EeV}$, which may be within the uncertainty in the energy determination for this event [39]. Furthermore, it was already noted that a positively charged particle injected by the nextdoor galaxy M82 may have been deflected towards the arrival direction of the highest energy event [41]. Note that although M82 is not a strong radio galaxy, it has been described as the archetypal starburst galaxy (supernova rate as high as $0.2-0.3 \mathrm{yr}^{-1}$ ) and as a prototype of superwind galaxies. In this picture, the central region of the galaxy harbors a population of heavy ions (mainly produced in supernova explotions) that could be re-accelerated to ultra high energies at the terminal shocks of galactic superwinds generated by the starburst. It is noteworthy that M82 could also be responsible of the "super-GZK" Yakutsk event. In addition, it is interesting to point out that the regular component of the galactic magnetic field acts as a giant lens for nuclei where $E / Z \leq 50 \mathrm{EeV}$, yielding significant deflections of CR trajectories [42].

Finally, we would like to stress another remarkable feature of nucleus primaries. It was already noted that nuclei heavier than iron could be accelerated to super-high energies [43]. Moreover, the dominant mechanism for energy losses in the bath of the universal cosmic radiation is the photodisintegration process, which was studied in detail for the case of ${ }^{197} \mathrm{Au}$ (see Fig. 3). In particular, the photodisintegration rate $R$ of a gold nucleus with Lorentz factor $\gamma_{0}=1 \times 10^{9}\left(\gamma_{0}=2 \times 10^{9}\right)$ is $R=3.22 \times 10^{-15} \mathrm{~s}^{-1}\left(R=1.55 \times 10^{-14} \mathrm{~s}^{-1}\right)$,

2 It should be noted, that the photodisintegration history of the nucleus was performed using the single/double-nucleon emission energy threshold of ref. [39]. The value of $A$ is thus slightly different to that of Ref. [40], because the increase of the threshold energy lengthens the propagation distance. 
and the energy loss time is defined by

$$
\tau^{-1} \equiv \frac{1}{E} \frac{d E}{d t}=\frac{1}{\gamma} \frac{d \gamma}{d t}+\frac{R}{A}
$$

The energy loss due to photopair production could be estimated to be roughly $Z^{2} / A$ times higher than that of a proton with the same Lorentz factor, i.e., for $\gamma_{0} \approx 10^{9}, \tau_{e^{+} e^{-}}^{-1} \equiv 1 / \gamma d \gamma / d t \approx 2 \times 10^{-18} \mathrm{~s}^{-1}$ [44]. For a gold nucleus then we get, $\tau \approx 6 \times 10^{16} \mathrm{~s}\left(\tau \approx 1 \times 10^{16} \mathrm{~s}\right)$. Now, using the previous formulae for $\tau$, it is straightforward to show that a gold nucleus emitted with a Lorentz factor $\approx 10^{9}$, after propagating $100 \mathrm{Mpc}$ still has an energy above $100 \mathrm{EeV}$. Specifically, gold nuclei injected with $197 \mathrm{EeV}$, will mutate to $A \approx 166-$ 168 with an energy $\approx 167 \mathrm{EeV}$, whereas for $E_{0}=394 \mathrm{EeV}$, the surviving fragment would have $A=89-91$ with an energy $\approx 180 \mathrm{EeV}$. Note that both values are within 1 standard deviation of the highest energy event recorded by AGASA $(E=210 \mathrm{EeV}$, whose accuracy on energy determination is $30 \%$ [7]). Furthermore, in this case the deflections of the CR trajectories would be enough to conceal the source location. The above valuation together with our previous analysis suggest that elements heavier than iron are likely to generate some of the "super-GZK" events. It perhaps should be interesting to try to simulate showers initiated by super-heavy nuclei considering the internal nuclear structure, specially when the hadonic interactions are modelled with SIBYLL.

In summary, nucleus induced showers are in complete agreement with the experimental data reported by the groups of Fly's Eye, Yakutsk and AGASA. Moreover, if the highest energy CRs are heavy nuclei, one cannot yet rule out that extragalactic/galactic magnetic fields could tangle up the particle paths, camouflaging the exact location of sources. The astrophysics parameters governing CR propagation are still somewhat uncertain, and better statistics on the arrival directions, energy spectra and composition are indispensable for understanding the origin of the highest energy particles ocurring in nature. Nonetheless, it does not seem reasonable to invoke "top-down" models to explain the existing data since they would fail to explain the nucleus-component, certainly present at the end of the spectrum.

\section{Acknowledgments}

Special thanks go to Gabriela Anchordoqui for carefully extracting the experimental data from Fig. 3 of Ref. [5]. The work was supported by CONICET (Argentina), and the National Science Foundation. 


\section{References}

[1] A. A. Penzias and R. W. Wilson, Astrophys. J. 142 (1965) 419.

[2] K. Greisen, Phys. Rev. Lett. 16 (1966) 748; G. T. Zatsepin, and V. A. Kuz'min, Pis'ma Zh. Éksp. Teor. Fiz. 4 (1966) 114 [JETP Lett. 4, 78 (1966)].

[3] For a recent survey see for instance, S. Yoshida and H. Dai, J. Phys. G 24 (1998) 905.

[4] M. A. Lawrence, R. J. O. Reid and A. A. Watson, J. Phys. G 17 (1991) 733.

[5] D. J. Bird et al., Astrophys. J. 441 (1995) 144.

[6] N. N. Efimov, N. N. Efremov, A. V. Glushkov, I. T. Makarov, and M. I. Pravdin, in Astrophysical Aspects of the Most Energetic Cosmic Rays, (eds. M. Nagano, F. Takahara, World Scientific 1991), p.434. See also, E. E. Antonov et al., Pis'ma Zh. Éksp. Teor. Fiz. 69 (1999) 614 [JETP Lett. 69, 650 (1999)].

[7] M. Takeda et al., Phys. Rev. Lett. 81 (1998) 1163; astro-ph/9902239.

[8] R. J. Protheroe, A. P. Szabo, Phys. Rev. Lett. 69 (1992) 2885.

[9] C. A. Norman, D. B. Melrose, and A. Achtenberg, Astrophys. J. 454 (1995) 6.

[10] E. Boldt and P. Gosh, Mon. Not. R. Astr. Soc. 307 (1999) 441; E. Boldt and M. Loewenstein astro-ph/0006221.

[11] C. Cesarsky and V. Ptuskin, Proc. 23rd Int. Cosmic Ray Conf. (Calgary) 2 (1993) 341.

[12] E. Waxman, Phys. Rev. Lett. 75 (1995) 386. This hypothesis, however, is not free of problems; F. W. Stecker, Astropart. Phys. (to be published) astroph/9911269; S. T. Scully and F. W. Stecker astro-ph/0006112.

[13] J. P. Rachen and P. L. Biermann, Astron. Astrophys. 272 (1993) 161.

[14] P. Bhattacharjee, and G. Sigl, Phys. Rep. 327 (2000) 109, and references therein.

[15] F. W. Stecker, Astrophys. J. 157 (1969) 507.

[16] F. Halzen, R. A. Vázquez, T. Stanev, and H. P. Vankov, Astropart. Phys. 3 (1995) 151.

[17] R. J. Protheroe and P. L. Biermann, Astropart. Phys. 6 (1995) 45; erratum ibid. 7 (1997) 181.

[18] G. Sigl, K. Jedamzik, D. N. Schramm and V. Berezinsky, Phys. Rev. D 52 (1995) 6682.

[19] G. Sigl, S. Lee, D. N. Schramm, and P. S. Coppi, Phys. Lett. B 392 (1997) 129.

[20] J. W. Elbert and P. Sommers, Astrophys. J. 441 (1995) 151. 
[21] J. L. Puget, F. W. Stecker and J. H. Bredekamp, Astrophys. J. 205 (1976) 638.

[22] L. A. Anchordoqui, Phys. Rev. D 61 (2000) 087302, and references therein.

[23] V. S. Berezinsky, S. V. Bulanov, V. A. Dogiel, V. L. Ginzburg, and V. S. Ptuskin, Astrophysics of Cosmic Rays, (North Holland, 1990). See in particular Fig. 4.17, p.142.

[24] J. Linsley, Astrophys. J. 235 (1980) L167. A dust grain does not seem to fit the highest energy event either; L. A. Anchordoqui, M. T. Dova, T. P. McCauley, T. Paul, S. Reucroft and J. D. Swain, asto-ph/0006071.

[25] R. M. Baltrusaitis et al., Nucl. Inst. Methods Phys. Res., Sect. A 240 (1985) 410.

[26] A. Capella, U. Sukhatme, C. I. Tan and J. Tran Thanh Van, Phys. Rep. 236 (1994) 225.

[27] A. B. Kaidalov, Phys. Lett B 116 (1982) 459; A. B. Kaidalov and K. A. TerMartirosyan, Phys. Lett. B 117 (1982) 247; Yad. Fiz. 39 (1984) 1545 [Sov. J. Nucl. Phys. 39, 979 (1984)].

[28] T. Regge, Nuovo Cimento 14 (1959) 951; V. N. Gribov, Sov. Phys. JETP 26 (1968) 414.

[29] This leading Regge trajectory with vacuum quantum numbers was originally proposed by G. F. Chew and S. C. Frautchi, Phys. Rev. Lett. 7 (1961) 394.

[30] T. Sjöstrand, Int. J. Mod. Phys. A 3 (1988) 751.

[31] N. N. Kalmykov, S. S. Ostapchenko, A. I. Pavlov, Nucl. Phys. B (Proc. Supp.) B52 (1997) 17. Details on hadron-nucleus interactions as described by QGSJET are discussed in, A. B. Kaidalov, K. A. Ter-Martirosyan and Yu. M. Shabel'skii, Yad. Fiz. 43 (1986) 1282 [Sov. J. Nucl. Phys. 43, 822 (1986)].

[32] R. S. Fletcher, T. K. Gaisser, P. Lipari and T. Stanev, Phys. Rev. D 50 (1994) 5710 .

[33] L. A. Anchordoqui, M. T. Dova, L. N. Epele and S. J. Sciutto, Phys. Rev. D 59 (1999) 094003.

[34] S. J. Sciutto, in Proc. XXVI International Cosmic Ray Conference, (Edts. D. Kieda, M. Salamon, and B. Dingus, Salt Lake City, Utah, 1999) vol.1, p.411.

[35] For details of the geomagnetic field see, A. Cillis and S. J. Sciutto, J. Phys. G 26 (2000) 309.

[36] For a concise review see the probability and statistics sections of, R. M. Barnett et al. (Particle Data Group), Phys. Rev. D 54 (1996) 155.

[37] N. Inoue et al. (AGASA Collaboration), in Proc. XXVI International Cosmic Ray Conference, (Edts. D. Kieda, M. Salamon, and B. Dingus, Salt Lake City, Utah, 1999) vol.1, p.357. 
[38] T. Wibig and A. W. Wolfendale, J. Phys. G 25 (1999) 1099.

[39] F. W. Stecker, and M. H. Salamon, Astrophys. J. 512 (1999) 521.

[40] L. N. Epele and E. Roulet, J. High Energy Phys. 10 (1998) 009.

[41] L. A. Anchordoqui, G. E. Romero and J. A. Combi, Phys. Rev. D 60 (1999) 10300.

[42] D. Harari, S. Mollerach and E. Roulet, J. High Energy Phys. 08 (1999) 022.

[43] L. A. Anchordoqui, M. T. Dova, T. P. McCauley, S. Reucroft and J. D. Swain, Phys. Lett. B 482 (2000) 343.

[44] G. Blumenthal, Phys. Rev. D 1 (1970) 1596; M. J. Chodorowski, A. A. Zdziarski, and M. Sikora, Astrophys. J. 400 (1992) 181. 


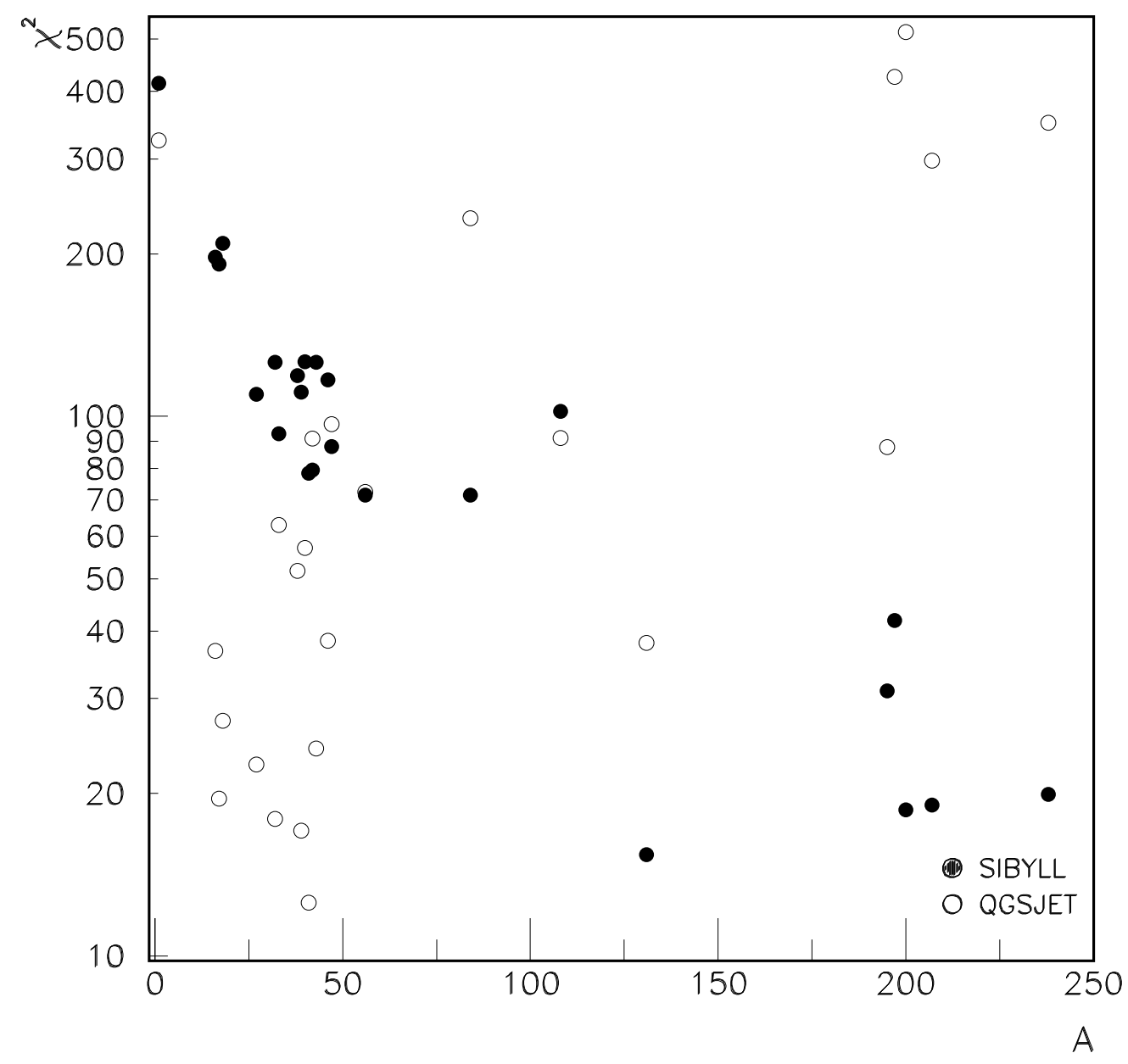

Fig. 1. Behavior of $\chi^{2}$ as a function of number of nucleons $A$. 


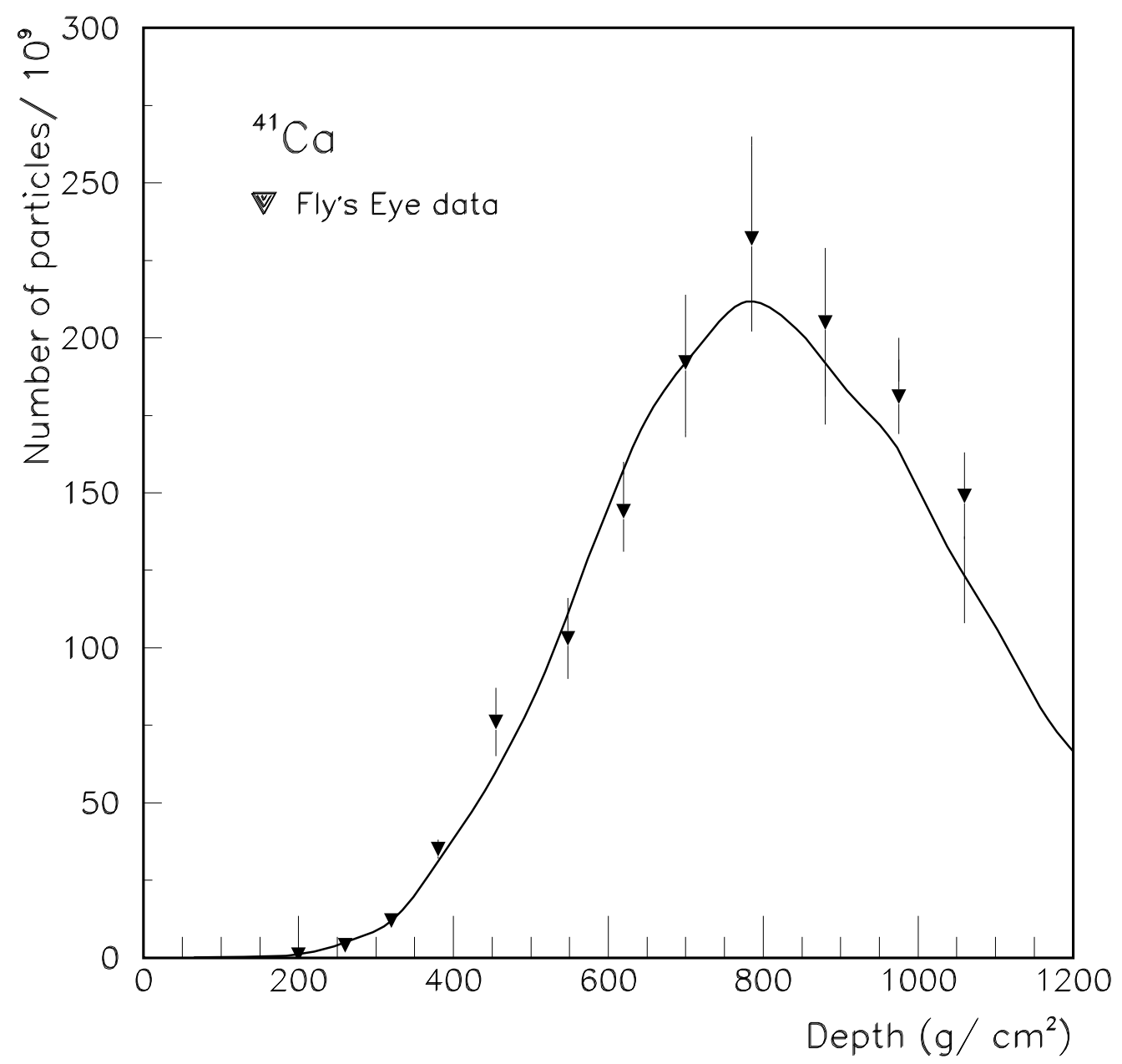

Fig. 2. Atmospheric cascade development of a $300 \mathrm{EeV}$ nucleus induced shower, superimposed over the Fly's Eye data. 


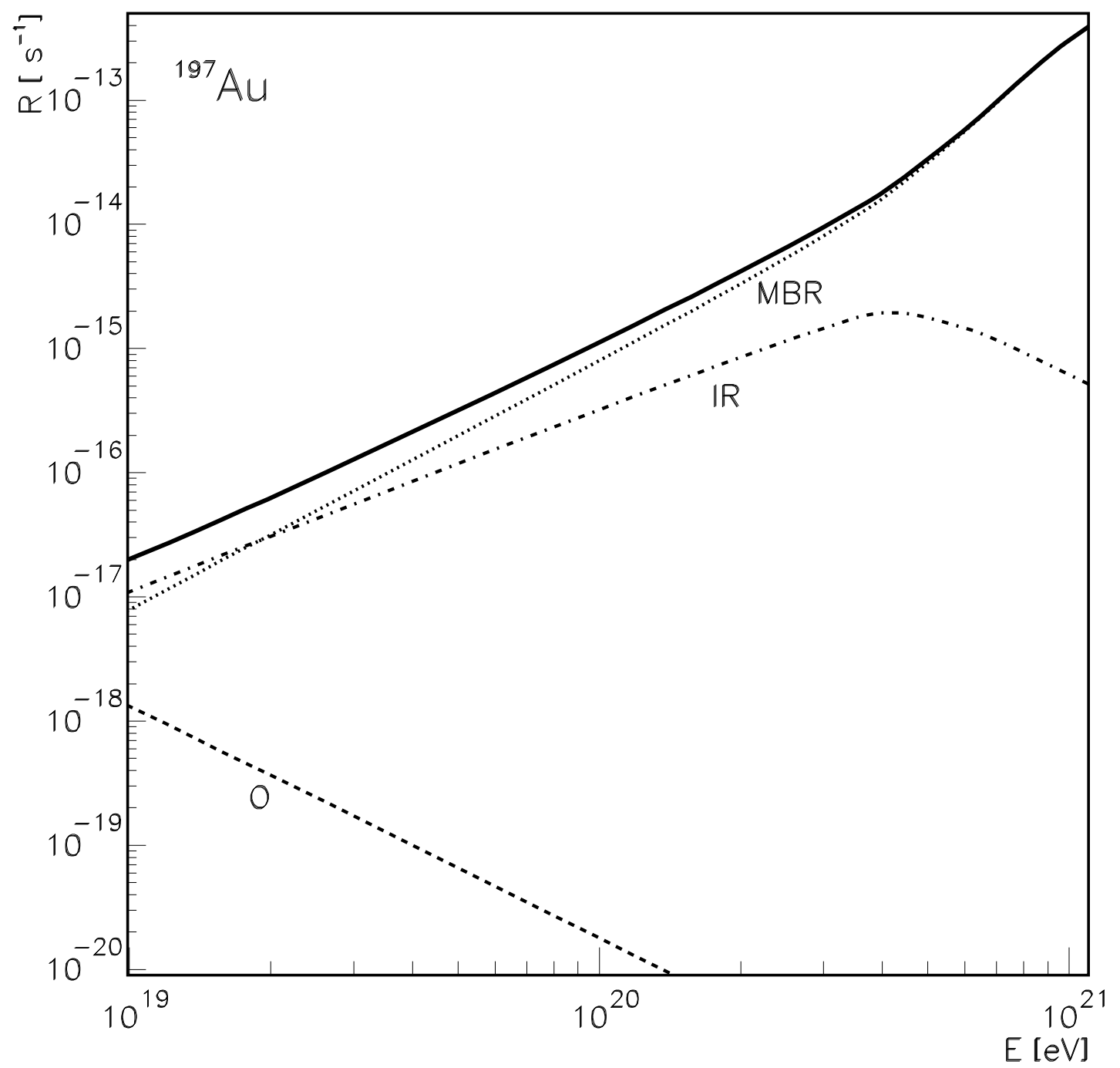

Fig. 3. Photodisintegration rate of ${ }^{197} \mathrm{Au}$ on $\mathrm{MBR}$, infrared radiation (IR), optical radiation $(\mathrm{O})$, as well as the total (solid line). This figure was originally published in Ref. [43] 